\title{
The expected second wave of COVID-19; predication based on previous pandemic
}

\author{
Misbahud Din ${ }^{1}$ and Madiha Asghar ${ }^{1}$ \\ ${ }^{1}$ Quaid-i-Azam University
}

August 6, 2020

\begin{abstract}
As the world past the few months of the deadly COVID-19 pandemic, the number of new cases continues to rise in several countries. By July 15, the confirmed COVID-19 cases reached 13,119,239, with 573,752 confirmed deaths worldwide.
\end{abstract}

\section{The expected second wave of COVID-19; predication based on pre- vious pandemics}

\author{
Madiha Asghar, Misbahud Din \\ Department of Biotechnology, Quaid-i-Azam University Islamabad, Pakistan \\ Corresponding author: MA (madihaasghar315@gmail.com)
}

As the world past the few months of the deadly COVID-19 pandemic, the number of new cases continues to rise in several countries. By July 15, the confirmed COVID-19 cases reached 13,119,239, with 573,752 confirmed deaths worldwide. ${ }^{3}$

There has been much speculation about if and when the second wave will crash down the earth and whether it will be more severe than the first crest. Based on the past pandemic experiences, the virus can return with even more lethal force, hence it is assumed that second wave of the pandemic could be more lethal. Epidemiologist assumed that COVID-19 is a new virus and it is not clear that either this pandemic will see second wave or not. They are studying past models to predict the coronavirus pattern and causes but the virus is novel, has its own viral behavior. ${ }^{4}$ It is also believed that the first wave will continue to spread with daily cases and deaths elevating and falling until the population attains herd immunity which seems unlikely without a vaccine widely available.

Over a century ago, a respiratory infection called as 1918 Spanish flu pandemic had far more devastating second wave and caused huge mass mortality. ${ }^{2}$ The second wave had been triggered by soldiers travelling across the areas, a probable virus mutation and the states failed to impose the physical distancing measures. Moreover, public frustration was the main cause in response to mandatory use of masks in 1918 that leads to protests for Anti-Mask League in San Francisco causing 45,000 confirmed flu cases with the deaths of over 3,000 individuals. Similarly, in Brazil, Spain, Germany and Argentina, hundreds of the people protested against stay at home orders being issued by the state and these protests caused the number of cases to bounce back. Currently, the mass protests in U.S, The Black Lives Matter, are under study to see if there will be a corresponding elevation in the cases. Same happened in Philadelphia in 1918, the massive parade celebrating the end of the war which resulted in huge outbreak. Recently, new outbreaks have reported in 
South Korea, Ireland, China, Germany and Singapore. The second wave will potentially overburden the healthcare sector.

Coronavirus spreads more easily from one person to another. In summer, people are more outdoor and able to stay apart which significantly decreases transmission but in winter, people will take more activities indoors and the virus can thrive in enclosed environments. Similarly, the schools and offices closure has also kept people apart, therefore, the reopening will also increase the risk of social gathering. Scientists have urged that the COVID-19 can last for more 3 years and can be halted when 60 to $70 \%$ of the world population is immune. ${ }^{1}$ As there is less chance that vaccines will be clinically available by this year, it is expected that the first wave of the pandemic will be followed by repetitive smaller waves that are likely to be appeared consistently. The waves are also expected to be geographically distinct and their intensity will depend on the regional control measures such avoiding social gathering. The worst scenario of larger second wave before the end of 2020 followed by smaller waves is expected. Such predications are based on the trends exhibited during influenza pandemic of 1918-19 which resulted about 50 million deaths, and 2009-10 pandemic of H1N1. ${ }^{2}$ Based on previous models, we must be prepared for at least 18-24 months of COVID-19 activity. With the wanes of pandemic, the SARS-CoV-2 is expected to continue circulation in the human population and synchronize to seasonal pattern decreased severity overtime, like other less pathogenic coronaviruses i.e. betacoronaviruses HKU1 and OC43 and influenza pandemics. ${ }^{1}$

In case of COVID-19, some fear that ease in restrictions and public ignorance regarding preventive measures may make the second wave of cases greater than the initial bursts. With the countries planning to ease restrictions imposed due to coronavirus but there is a concern about resurgence or second wave. Experts worry if the efforts of social distancing, wearing masks, hand washing and symptoms screening are not sustained, larger number of cases are likely to occur which will lead to reimposing self-quarantine polices. Some experts are hoping to gaze into the future by observing the behavior of previous respiratory illnesses. For example, influenza and common cold seem to be linked to the cool weather during the colder months. Same holds true for other types of seasonal coronaviruses and may also apply to SARS-CoV 2. The low humidity in the colder weather causes the virus to spread better. COVID-19 will be the part of future but whether the second wave will be a devastating tsunami or just a ripple may hinge on continued social distancing, masking, widespread testing, hospital preparation and vaccine development.

\section{References:}

1. Kissler, Stephen M., et al. "Projecting the transmission dynamics of SARS-CoV-2 through the postpandemic period." Science 368.6493 (2020): 860-868.

2. Saunders-Hastings, Patrick R., and Daniel Krewski. "Reviewing the history of pandemic influenza: understanding patterns of emergence and transmission." Pathogens 5.4 (2016): 66.

3. World Health Organization. 2020. https://www.who.int/news-room/ fact-sheets/detail/dengue-andsevere-dengue/. Accessed July 18, 2020.

4. Albery, G. F., Eskew, E. A., Ross, N., \& Olival, K. J. (2020). Predicting the global mammalian viral sharing network using phylogeography. Nature communications , 11 (1), 1-9. 